tion of modern film, and its mechanism is important to photographic manufacturers. Processing and some stages of the manufacture are only possible because of the ease of diffusion of ions and molecules in the medium into and out of gelatine. For special purposes, it is sometimes necessary either to harden or even insolubilize the gelatine, or to soften it, and gelatine can easily be modified in these ways. Gelatine has a profound effect on the sensitivity of the emulsions, and much work has been carried out on this; but the mechanism of the action is still not completely understood. It is desirable that the gelatine should be of uniform standard from batch to batch, particularly with respect to viscosity and sensitizing power.

\section{CAUSATION AND EXPLANATION IN THEORETICAL BIOLOGY}

IN a recent issue of the British Journal for the I Philosophy of Science (1, No. 4; February 1951), J. S. Wilkie considers some of the classical problems of explanation in biology in the light of a relative theory of causation. A cause is taken to be some factor to which is attributed the disturbance of some regularity. Living things are extremely complex systems in which the parts have causal relations inter se: the regular rhythm of the heart is disturbed by nervous stimulation; the regularity of an undifferentiated field of competent ectoderm is disturbed by the presence of an optic vesicle near some part of the field. But living things as relatively isolated systems can themselves be considered as complex regularities which are disturbed by environmental interference. Reaction with the environment can be considered as constituting a causal event only if there is some assignable disturbance of some regularity of the system : since the system is not totally isolated, it will have regular and constant relations with its environment which are necessary to its maintenance as a system; thus the entry of oxygen into the system cannot as such be considered as a causal relation with the environment. On the other hand, episodic changes such as a temporary increase or diminution of oxygen in the immediate environment definitely disturb the rhythms of the organism and can thus be considered as causal. Among such episodic causal events are the reactions of environmental changes with the sense-organs.

Whatever can be deduced from the known properties of the system alone receives explanation, but not causal explanation.

Mnemic phenomena introduce causal events of peculiar interest. With respect to the organism they are causal, since there is nothing in the structure of the organism from which we can deduce the memories it acquires. Whereas in reflex activity the organism behaves as an elastic system, in memorizing it behaves as an inelastic system. Mnemic traces, considered as imposed upon the structure common to a species, are consequences of causal events and therefore causal themselves with respect to the specific regularity of the species. But mnemic traces do not rernain indifferent to one another, as do the traces left in the wood of a tree by wet and dry seasons; they become organized inter se. Thus the behaviour of these traces has its own regularities which, since they are regularities, might be explained by a knowledge of the properties of the materials of the system (as cleavage patterns can be explained by molecular forces). This, however, appears improb. able, since the physical language lacks the necessary concepts (constructs) under which the peculiar behaviour of mnemic traces could be subsumed. Physical attractions all appear as functions of space and time, whereas mnemic attractions appear as functions of space, time and similarity.

Wilkie also attempts to justify teleological explanations; but it is not considered appropriate to treat these as causal explanations.

\section{FORESTRY COMMISSION ANNUAL REPORT FOR 1949}

7 HE thirtieth annual report of the Forestry Commissioners covering the year ending Sep. tember 30,1949 , has recently been published*. Since the passing of the Forestry Bill in 1919, the Commission has published quinquennial reviews of the work done, in 1924, 1929 and 1934. The Second World War brought the gap to fifteen years, the present report being the first to be issued since the War ended.

In the thirty years of its existence the Forestry Commission has acquired an area of 1,500,000 acres, of which 971,000 were classified at the time of acquisition as plantable. In addition, 57,000 acres of standing woodland have been acquired. By the aid of planting grants, private owners planted 182,000 acres. Planting operations were of necessity severely interrupted during the Second World War.

With regard to the plantations, which range from 1 to 30 years in age, there is a total of 550,624 acres, of which 515,666 are conifers and 34,958 hardwood trees. The youngest ones, $1-5$ years old, cover the largest area $(123,483$ acres $)$, while the oldest, 26-30 years, the smallest $(32,959$ acres). These latter are now producing some revenue. Furthermore, there is what the Commissioners term a by-product, namely, the establishment at a small cost of six national forest parks comprising 240,000 acres.

Other minor Forestry Acts have been passed since 1919 : the Act (Transfer of Woods) of 1923, which empowered the transfer of the majority of the existing Crown woods-for example, the New Forest, Forest of Dean - to the Commission, and in $1927 \mathrm{a}$ short Act authorizing an increase in the number of Commissioners and empowering them to make bylaws with respect to their properties. The issue in 1943 of a report by the Commissioners on post-war forest policy resulted in the Forestry Act of 1945. The Act brought into force ministerial responsibility for future forest policy, an action which was by then very justifiable. The Minister for Agriculture and Fisheries was responsible for England and Wales, while Scotland came under the Secretary of State, the lands acquired since 1919 being invested under the appropriate Minister.

A further measure of the Forestry Act of 1947 provided for the dedication of private woodland to forestry purposes, and dealt with such matters as the enforcement of dedication covenants in England and Wales and of dedication agreements in Scotland. This measure proved very contentious, and has been subject to considerable modifications.

Lastly, a New Forest Act, 1949, was passed just after the period of this report. The Act amends the

* Forestry Commission. Thirtieth Annual Report of the Forestry Commissioners for the Year ending September 30th, 1949. Pp.
15 plates. (London: H.M. Stationery Offlce 1950.) 48. net. 
contribution of the verderers and gives them certain additional powers. Provision was also made to enable the verderers to authorize the enclosure of additional land up to a maximum of five thousand acres, for the purpose of growing timber, and the enclosure of a maximum of three thousand acres for cultivation and improvement of grazing. The long-standing problem of the ancient and ornamental woods was met by providing that the verderers may authorize the Commissioners, subject to certain conditions, to make enclosures, individually not exceeding twenty acres in area, for the rehabilitation of these woods.

\section{RADIO-WAVE PROPAGATION AT VERY HIGH FREQUENCIES}

$T$

HE radio communication engineer is continually seeking opportunities of using the shorter wavelength portions of the radio spectrum for services and applications of various types. Reference was made recently in Nature (April 21, p. 617) to the fact that the report of the Beveridge Committee on broadcasting in Great Britain directed attention to the need for actively developing the use of fre. quencies above $30 \mathrm{Mc} / \mathrm{s}$. (wave-lengths shorter than $10 \mathrm{~m}$.) in order to relieve the congestion in Europe in the medium- and long-wave bands, and to provide a greater diversity in the broadcasting programmes available in Great Britain. The present British television service is being developed in the frequency range 4l-68 Mc./s., while a band of frequencies in the region of $90 \mathrm{Mc}$./s. is available for the development of sound broadcasting.

It was thus very timely that, at a meeting of the Radio Section of the Institution of Electrical Engineers held on April 11, three papers were read describing the results of recent investigations on the propagation of waves at certain very high frequencies. The first of these, entitled "A V.H.F. Field-Strength Survey on 90 Mc./s.", by H. L. Kirke, R. A. Rowden and $G$. I. Ross, describes measurements made by staff of the British Broadcasting Corporation on the radiation from an experimental transmitter at Wrotham in Kent. Automatic field-strength recording equipment was installed in a vehicle which cruised at a speed of about $1 \mathrm{~m}$.p.h. over a distance of from 50 to $500 \mathrm{yd}$. in the neighbourhood of each point selected for observation. In this manner measurements were made in various directions from the transmitter up to distances of some 60 miles, which includes the whole of the London area and a considerable portion of south-east England.

It was found that, while the field-strength varied markedly with small changes in position in a built-up area or along a tree-lined road, useful and consistent results could be obtained from the averages of the recorded readings. Thus, it was shown that buildings of normal height in London reduce the average received field by 10-12 db.; but the average for one street may be taken as the average for the district to an accuracy of about $\pm 4 \mathrm{db}$. The variation of field-strength along the street is less with horizontal than with vertical polarization. In hilly country, the minimum field-strength occurred on the near-side slope of a valley and not at the lowest point. On rising ground facing the transmitter, horizontally polarized waves gave stronger fields than vertical polarization; but, when once the peak of the hill has been passed, there is a range on the far-side slope in which vertically polarized radiation gives the stronger field, a fact which confirms the results of previous investigators. From all the results obtained in this work a predicted field-strength contour map has been prepared and is included in the paper. This map refers to the power $(25 \mathrm{~kW}$.) and aerial height (500 ft.) of a B.B.C. transmitting station now in experimental use at Wrotham. This investigation has shown that when the Wrotham station is brought into full operation, it is likely to gave a satisfactory broadcasting service, defined by a field-strength of $2 \mathrm{mV} . / \mathrm{m}$. or more, to Greater London and most of south-east England.

The other two papers read at the meeting were under the common title of "The Propagation of Metre Radio Waves beyond the Normal Horizon" and describe some theoretical and experimental investigations carried out during the past few years by staff of the Radio Division of the National Physical Laboratory. In Part 1, Dr. J. A. Saxton considers the propagation of very-high-frequency waves over land, and discusses the relative importance of changes in atmospheric refraction near the surface of the earth and the reflexion of waves from highlevel inversion layers. Using information on typical atmospheric conditions at various heights, obtained from the usual meteorological charts, the author presents some useful sets of graphs showing the field-strength to be expected at various distances up to $400 \mathrm{~km}$. (250 miles) from the transmitter. The comparative influence on the received field of the three main factors-simple atmospheric refraction, guiding of the waves in a low-level duct, and reflexion from inversion layers-is well illustrated by these graphs. Consideration is also given to the effects of scattering of radio energy by turbulent eddies in the atmosphere; but, except at distances exceeding about $250 \mathrm{~km}$., it appears that such scattering is of less importance than the effect of the mechanisms of abnormal refraction, which may be deemed to include reflexion at elevated inversion layers.

The third paper, forming Part 2 of the joint contribution, is entitled "Experimental Investigations at Frequencies of 90 and $45 \mathrm{Mc}$./s.", by Dr. J. A. Saxton, G. W. Luscombe and G. H. Bazzard. This describes the results of measurements of the fieldstrength on these frequencies over distances such that direct transmission from the sender to the receiver was impossible. Two paths, of lengths 110 and $270 \mathrm{~km}$., have been investigated on $90 \mathrm{Mc} . / \mathrm{s}$., and one of $160 \mathrm{~km}$. at $45 \mathrm{Mc}$. $/ \mathrm{s}$. The recorded fieldstrengths varied over a range of some $40 \mathrm{db}$., and there is some indication of a diurnal variation. The statistical distribution of quasi-peak field-strengths as a function of time has been determined, and it is shown that values of field considerably in excess of those corresponding to standard atmospheric refraction often occur. An attempt is made in the paper to interpret the observed field-strengths in terms of the meteorological data obtained from the routine radio sounding-balloon ascents made in Great Britain, and some degree of correlation has been obtained. The results of such investigations are of considerable importance in planning radio com. munication services for operation over distances well in excess of the normal optical range, and also in enabling an assessment to be made of the extent of any mutual interference likely to be experienced between broadcasting and other services operating in the same frequency channel. 